\title{
Efficient and rigorous modeling of second-harmonic generation including moderate pump-depletion
}

\author{
Björn Maes, Peter Bienstman, Roel Baets \\ Department of Information Technology, Ghent University - IMEC, St.-Pietersnieuwstraat 41, 9000 Ghent, Belgium \\ bjorn.maes@intec.ugent.be
}

\begin{abstract}
We present an efficient modeling method to simulate second-harmonic generation in arbitrary two-dimensional devices. By employing eigenmode expansion the algorithm is fully vectorial and especially suited for periodic structures. We include moderate pump-depletion using iteration.

(C)2005 Optical Society of America

OCIS codes: (190.2620) Frequency conversion; (230.4320) Nonlinear optical devices
\end{abstract}

\section{Introduction}

Second-harmonic generation ( $\mathrm{SHG}$ ) has many appealing applications. Among them is the possibility to create efficient sources at frequencies where direct lasers are lacking. Furthermore cascading processes (consecutive upand down-conversions) can impart phase changes that are used for all-optical switching purposes.

A promising research route is the combination of these second-order effects with high-contrast periodic structures or photonic crystals. These wavelength-scale components convey new possibilities for dispersion and field confinement. Many studies and experiments focus on linear two-dimensional (2D) devices. Consequently there is a need for numerical tools that make it possible to examine these structures for nonlinear functionalities.

Previous work mainly explored the one-dimensional plane-wave or single-mode case for quasi-phase matching structures [1]. Tools for 2D components are developed recently, but they extend the BPM or FDTD algorithm [2,3]. As a result these methods need a grid for the entire structure, which increases the computational time and memory requirements.

In this work we extend the eigenmode expansion algorithm. This modal framework describes the fields as a sum of modes, that are determined using the transversal index profile. Only a few tens of eigenmodes are sufficient for rigorous and vectorial representation of the fields in most components. Because of this effective representation and the fact that no grid is introduced, the method proves very efficient.

Since we include higher-order and radiation modes we are able to completely model confinement effects and radiation losses. These losses are absorbed at the edges of the computational domain by PML boundary conditions. The method is especially useful for periodic structures as we can effectively reuse data, such as mode propagation constants, overlap integrals, etc. Furthermore, physical insight is gained because we have immediate access to the particular mode contributions.

Pump-depletion effects offer new device functionalities and we include them in an approximate way. After a few iterations the fundamental and second-harmonic calculations converge on a depleted solution, that is accurate up until about 10\% pump-depletion.

\section{Method}

The algorithm is a combination of the linear scattering matrix scheme and the well-known nonlinear coupled-mode equations. As already mentioned the field is handled as a superposition of modes. Therefore, once the modes are determined, the object is to calculate their (complex) amplitudes. These amplitudes, that are bundled in vectors, depend on the position along the propagation direction. It turns out that these vectors relate to each other via matrix operations [4]. Because a limited number of modes are sufficient, these matrix manipulations are efficient, and one can determine the output fields in only one multiplication using the input field vectors.

In mode expansion the structure is divided in invariant sections along a main propagation direction, with each section having its distinct set of eigenmodes. The scattering matrix for an entire structure is built from the matrices of the individual sections and the interfaces. Interfaces between different sections are handled with the known mode-matching method, that imposes continuity of the tangential field components [4].

The calculations for the fundamental frequency (FF) and the second harmonic ( $\mathrm{SH}$ ) are largely separate. Each frequency has its own amplitude vectors and scattering matrices. The general form for both frequencies is the same however, and is the following: 


$$
\left[\begin{array}{l}
F_{\text {out }} \\
B_{\text {out }}
\end{array}\right]=S\left[\begin{array}{l}
F_{i n} \\
B_{\text {in }}
\end{array}\right]+\left[\begin{array}{l}
N_{f w} \\
N_{b w}
\end{array}\right]
$$

The vectors are shown in Fig. 1(a). $S$ denotes the total $(2 n) \mathrm{x}(2 n)$ scattering matrix, with $n$ the number of modes. $N_{f w}$ and $N_{b w}$ are $n$-element vectors, that represent the nonlinear modification of the linear scheme. The coupling between the frequencies happens through the determination of these vectors. As an example, if there is no external SH input, we are often interested in the SH $N_{f w}$ and $N_{b w}$, as it determines the conversion efficiency.

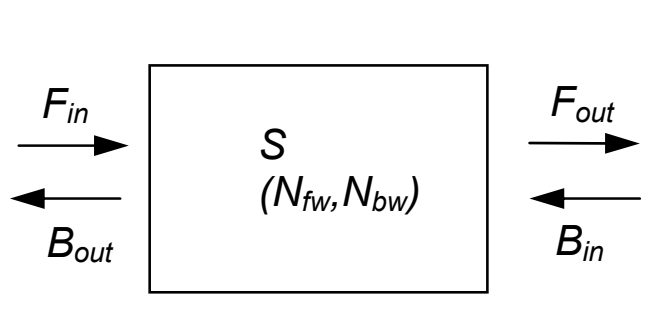

(a)

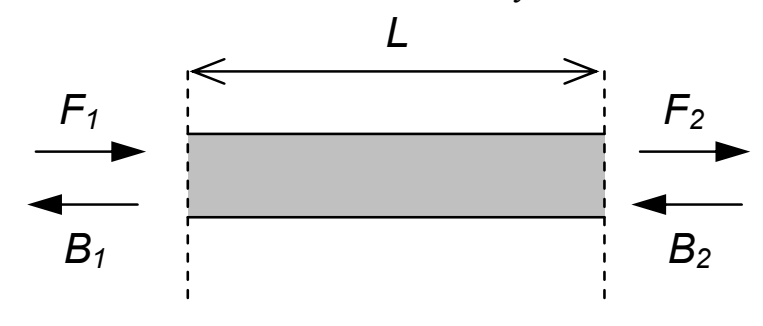

(b)

Fig. 1. Amplitude vector positions in the case of: (a) a global device, and (b) a single invariant section.

For a single section, see Fig. 1(b), we obtain:

$$
\left[\begin{array}{l}
F_{2} \\
B_{1}
\end{array}\right]=\left[\begin{array}{cc}
\operatorname{diag}\left(\exp \left(-j \beta_{i} L\right)\right) & 0 \\
0 & \operatorname{diag}\left(\exp \left(-j \beta_{i} L\right)\right)
\end{array}\right]\left[\begin{array}{l}
F_{1} \\
B_{2}
\end{array}\right]+\left[\begin{array}{c}
N_{f w} \\
N_{b w}
\end{array}\right],
$$

with $\operatorname{diag}()$ a diagonal matrix, $\beta_{i}$ the mode propagation constants and $L$ the section length. The determination of the nonlinear terms $N_{f w}$ and $N_{b w}$ is different but analogous for both frequencies. It involves an approximate solution of the coupled-mode equations. Only modes propagating in the same direction are considered to couple nonlinearly, because of phase-matching. Using the slowly-varying envelope approximation and mode orthonormality each amplitude is described by a first-order differential equation. For the SH nonlinear terms this equation is solved to obtain for mode $i$ :

$$
N_{i}^{S H} \propto \sum_{k l} O_{k l i} A_{k}^{F F}(0) A_{l}^{F F}(0) \frac{\exp \left(j \Delta \beta_{k l i} L\right)-1}{\Delta \beta_{k l i}},
$$

with $O_{k l i}$ overlap integrals between FF modes $(k, l)$ and SH mode $i . A_{k}^{F F}(0)$ and $A_{l}^{F F}(0)$ are the FF mode amplitudes at the beginning of the section. The phase-matching factor is governed by $\Delta \beta_{\mathrm{kli}}=\beta_{\mathrm{i}}{ }^{\mathrm{SH}}-\beta_{\mathrm{k}}{ }^{\mathrm{FF}}-\beta_{1}^{\mathrm{FF}}$. For these SH terms we assume that the FF amplitudes remain constant in the section, there is no depletion. If we use Eq. (1) in the coupled-mode equations for the FF however, depletion is incorporated into the $N^{F F}$-terms, albeit in an approximate way. Therefore the method remains accurate for weak to moderate depletion values. The exact expressions for the FF terms are very similar to Eq.(1), although they use different overlap integrals and the SH amplitudes.

If we ignore pump-depletion, the algorithm needs only one run: The FF is calculated linearly. We use these FF amplitudes for the SH generation terms (see Eq.(1)) to build the scattering matrices and immediately obtain the conversion efficiency. To include depletion, we iterate the FF and SH calculations, each time using the amplitudes of one frequency to refine the other frequency vectors. Usually about ten iterations are sufficient for convergence.

\section{Examples}

\subsection{Photonic crystal cavity}

Fabrication of high-quality, small-volume resonators in 2D photonic crystals has been achieved. This strong confinement may be exploited for efficient SHG. Therefore we designed a doubly-resonant device, shown in Fig.2(a). Because of periodicity the structure is ideal for our method. Furthermore, because the field is strongest in the cavity, only the center rod has to be considered nonlinear. For the operation of the device there are conditions that have to be fulfilled at both frequencies: occurrence of a bandgap, a waveguide mode and a cavity resonance. The geometry consists of rods with indices $n_{\mathrm{FF}}=3.5$ and $n_{\mathrm{SH}}=2.95$ in air, with diameter $0.5 a$. The waveguide rods and cavity rod measure $0.3 a$ and $0.7 a$, respectively. The $\mathrm{FF}$ resonance is tuned to $1 / 2$ of the SH resonance-frequency, by adjusting the center rod index to 3.5743 . 


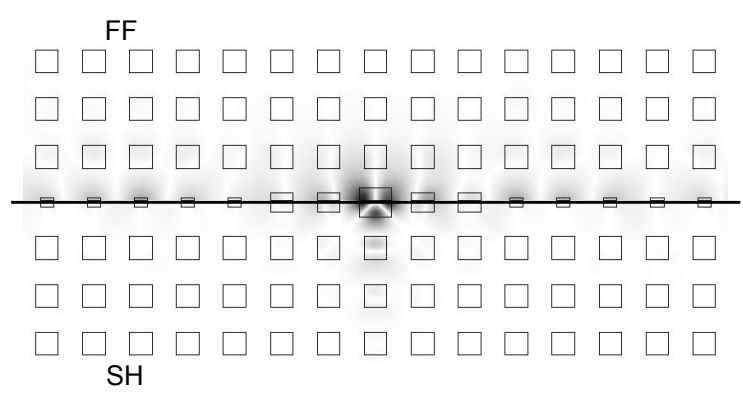

(a)

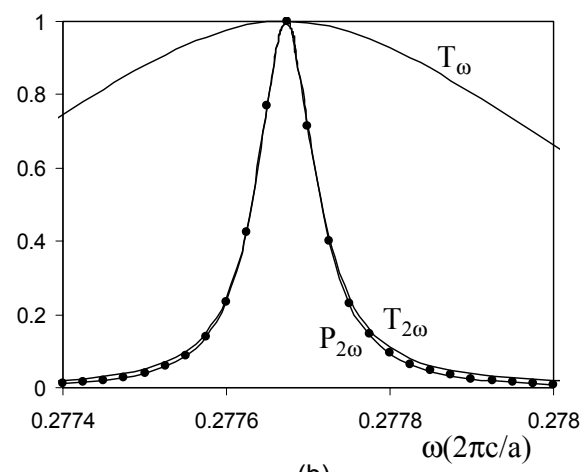

(b)

Fig. 2. (a) Geometry of the photonic crystal resonator device. Upper (lower) half shows the FF (SH) field on resonance. (b) $\mathrm{T}_{\omega}\left(\mathrm{T}_{2 \omega}\right)$ shows transmission of $\mathrm{FF}(\mathrm{SH})$. $\mathrm{P}_{2 \omega}$ depicts the normalized generated $\mathrm{SH}$ power. The SH frequency is halved.

The calculation results are shown in Fig. 2(b). The transmissions $T_{\omega}$ and $T_{2 \omega}$ (from the left to the right waveguide) indicate that the resonances are tuned. $\mathrm{P}_{2 \omega}$ is the generated SH power in the left (or right) waveguide, as the cavity is pumped from one side by the FF. Depletion is not included in these calculations. Only 30 modes were used at both frequencies. Moreover, we can choose which subsets couple nonlinearly. For the other, linearly coupled modes, we ignore the nonlinear terms in the $N$-vectors. In this example, allowing the 10 lowest order modes to couple nonlinearly is sufficient for accurate results.

\subsection{Deeply etched waveguide}

The waveguide with air slots is depicted in Fig. 3(a). Diffraction losses are an important issue here, and necessitate the inclusion of radiation modes. We have extended the study in [3] to devices with more periods. Because of efficiency and periodicity even the structure with 40 periods needs only minutes to calculate. The simulated SHG efficiency with and without depletion is depicted in Fig. 3(b).

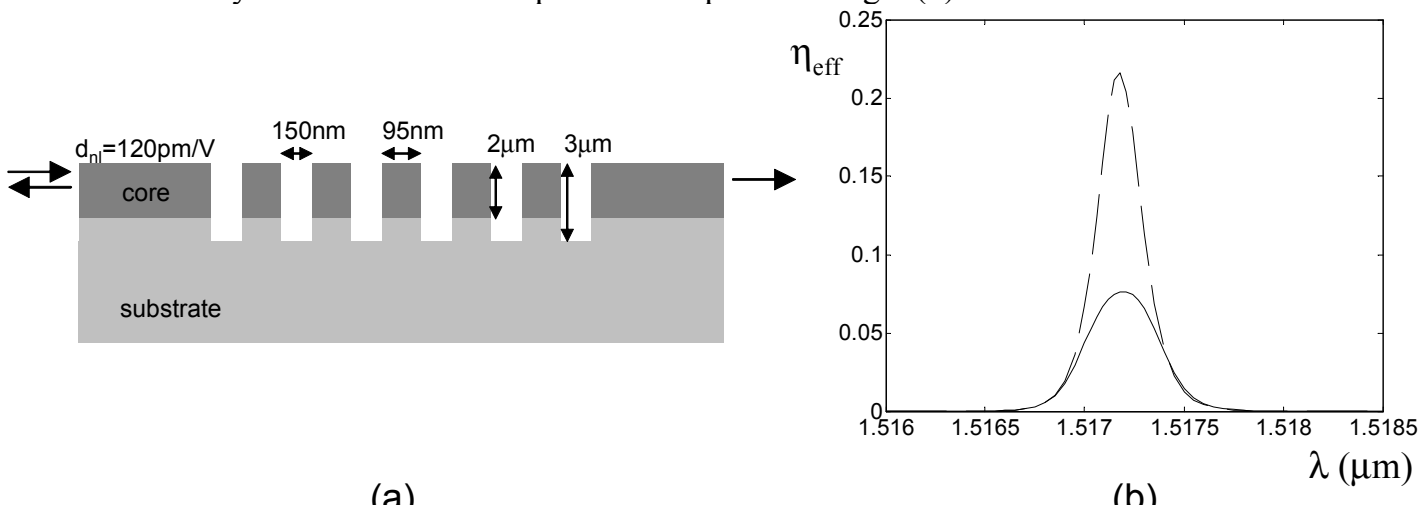

(a)

(b)

Fig. 3. (a) Geometry of the deeply etched waveguide. (b) SHG efficiency $\eta_{\text {eff }}$ versus fundamental wavelength for a 40period structure without (dashed line) and with (solid line) pump-depletion.

\section{Conclusion}

We presented an extension to the mode expansion method for rigorous simulation of SHG. Efficiency stems from the lack of a grid in the propagation direction, the need for a limited set of eigenmodes and the reuse of data in periodic structures. Because of the inclusion of higher-order modes effects such as bandgap-confinement and radiation losses are modeled effectively. The software will be freely available at http://camfr.sourceforge.net.

\section{References}

[1] Y. Jeong, B. Lee, "Matrix analysis for layered quasi-phase-matched media considering multiple reflection and pump wave depletion," IEEE Journal of Quantum Electronics, 35, 162-172 (1999).

[2] A. Locatelli, F.-M. Pigozzo, F. Baronio, D. Modotto, A.-D. Capobianco, C. De Angelis, "Bidirectional beam propagation method for secondharmonic generation in engineered multilayered waveguides," Optical and Quantum Electronics, 35, 429-452 (2003).

[3] Y. Dumeige, F. Raineri, A. Levenson, X. Letartre, "Second-harmonic generation in one-dimensional photonic edge waveguides," Phys. Rev. E, 68, 066617 (2003).

[4] P. Bienstman, R. Baets, "Optical modelling of photonic crystals and VCSELs using eigenmode expansion and perfectly matched layers," Opt. Quantum Electron. 33, 327-341 (2001). 


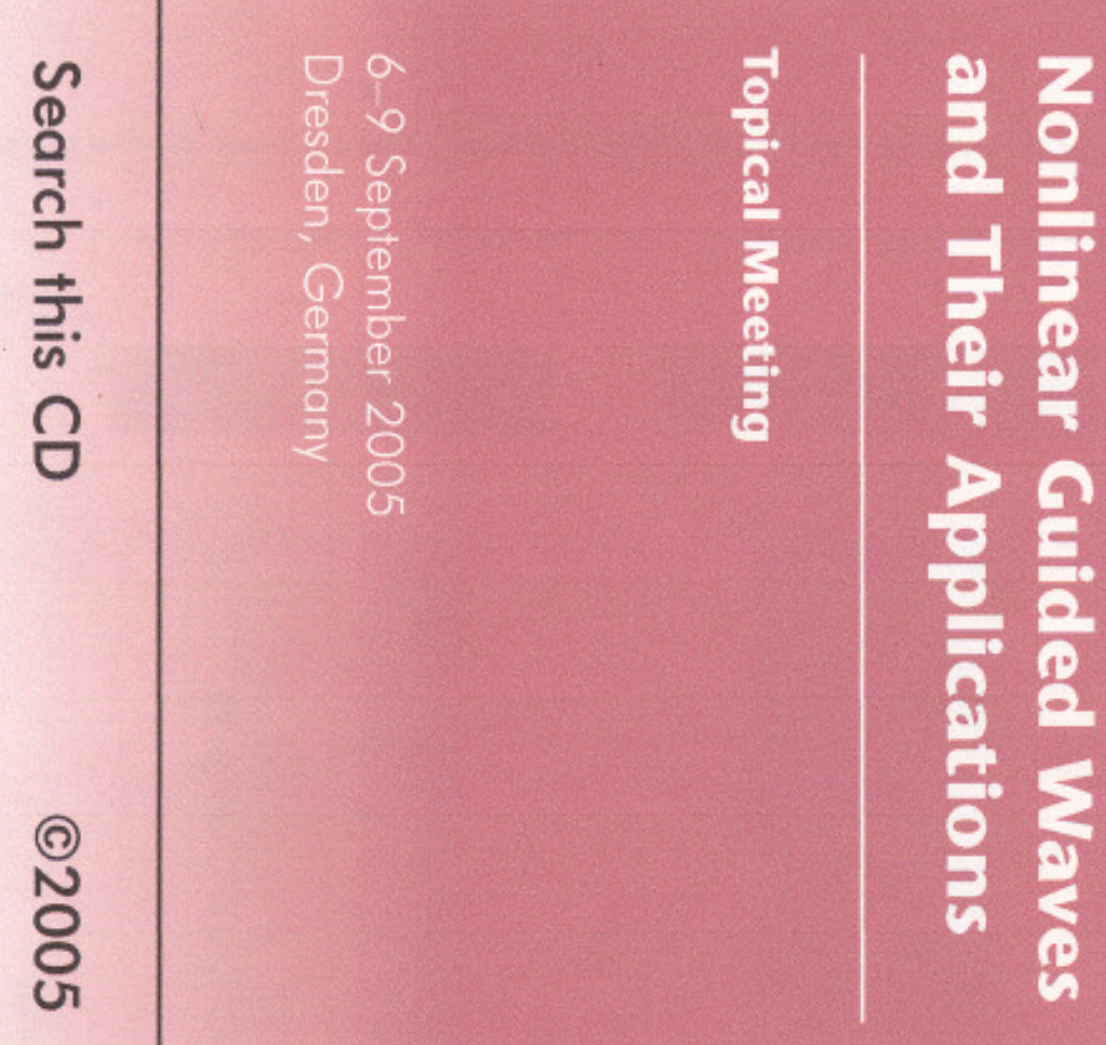

0
0
1
0
0
3
$\frac{1}{D}$
$\frac{1}{0}$

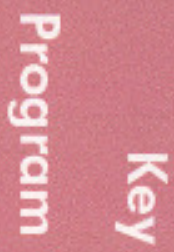

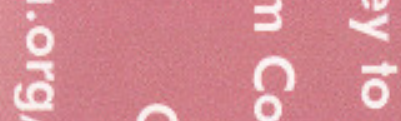

$\xi$
$\vdots$
$\vdots$
$\vdots$
$\vdots$
0
0

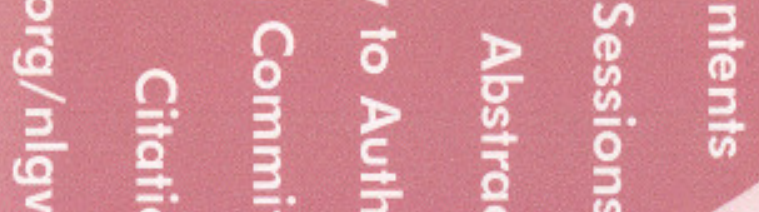

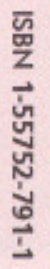

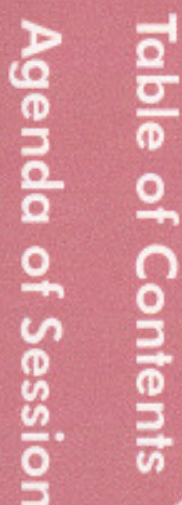

음 क

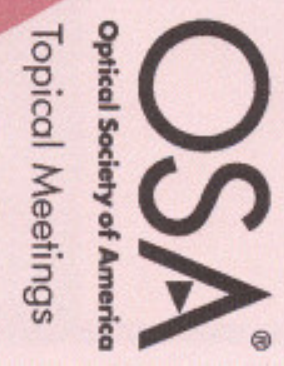

